\title{
ERPS TO SUBCLASSES OF NOUNS AND VERBS
}

\author{
Josef Petřek \\ Institute of Physiology, Faculty of Medicine, Palacký University, 77515 Olomouc, Czech Republic \\ e-mail:fesoj@tunw.upol.cz
}

Received: July 10, 2004; Accepted: September 4, 2004

Key words: ERPs/Word subclasses/Gender/Site of recording electrode

The present findings show the existence of significant differences in latency and amplitude of some waves of ERPs to three subclasses of nouns or verbs. Latency LP3 of ERPs to action verbs was shorter than latency of the same wave to abstract verbs. In nouns the relation was a similar (manipulable objects versus abstract ones). The amplitude of early positive components (P1, P2) and BN3 wave also depended on semantic attributes of nouns and verbs. Some waves of ERPs to motion verbs in our experiment had significantly higher amplitude than the same waves of ERPs to nonmanipulable objects. Also revealed was interaction between some ERP features and the subject's gender. It was primarily the amplitude of BP2, the size of which depended on gender in all cases - BP2 amplitude was significantly higher in females then in males. In the other components (BP1, BP4, BN2 and BN4) there were fewer significant differences and if they do occurred, then their amplitude was higher in males than in females. In some cases, gender also affected latency of waves LP2, LN1 and LN4 of ERPs to the same noun or verb subclass - latencies of these waves were shorter in females.

\section{INTRODUCTION}

Analysis of speech disorders in patients with a lesion in the central nervous system (see e.g. ${ }^{2-5,8,17}$, etc.) has brought clinical researchers to the conclusion that different functional systems of the brain participate in the language processing of nouns and verbs.

The conclusion of clinical researchers is confirmed by the findings of several elctrophysiological studies. Thus e.g. Teyler et al. ${ }^{18}$ found that nouns and verbs presented by sound produced a strong N100-P160 in both hemispheres. In application of the verbs, however, the latency of this wave in the record of the left hemisphere is shorter than in application of nouns. Brown at al. (1973) supports the findings of Teyler et al. The findings of Koenig and Lehmann $^{7}$ and Molfese et al. ${ }^{9}$ speak in favour of the as- sumption that different neuronal assemblies participate in the language processing of nouns and verbs.

Pulvermüller et al..$^{12-15}$ described the dynamics of the changes in electrical activity in various areas of both hemispheres under application of nouns evoking strong visual or sometimes motor association, and in application of verbs associated with motor activity. Action verbs produced maximal response over the anterior and central parts of the hemisphere, nouns in occipital sites - in greater detail see Pulvermüller et al. ${ }^{13}$. A later presentation by Pulvermüller et al. ${ }^{16}$ expanded the findings and made them more precise.

In this context it is necessary to be reminded of Kellenbach et al. ${ }^{6}$. In their paper the authors described the dynamics of changes in ERPs produced by nouns and verbs. When preparing the experiment they classified nouns and

Table 1. Multivariate analysis of variance: ERPs data

\begin{tabular}{|l|c|c|c|c|c|c|}
\hline & \multicolumn{6}{|c|}{ Table of all effects: ERPs data } \\
\hline Effect & Test & Value & F & Effect df & Error df & $\mathrm{p}$ \\
\hline Gender & Wilks & 0.587145 & 33.79 & 18 & 865.00 & 0.000000 \\
\hline Word subclass & Wilks & 0.760182 & 2.25 & 108 & 4964.54 & 0.000000 \\
\hline Electrode & Wilks & 0.256120 & 12.33 & 108 & 4964.54 & 0.000000 \\
\hline Gender $\times$ Word subclass & Wilks & 0.738741 & 2.49 & 108 & 4964.54 & 0.000000 \\
\hline Gender $\times$ Electrode & Wilks & 0.777569 & 2.06 & 108 & 4964.54 & 0.000000 \\
\hline Word subclass $\times$ Electrode & Wilks & 0.721663 & 0.44 & 648 & 14035.88 & 1.000000 \\
\hline Gender $\times$ Word subclass $\times$ Electrode & Wilks & 0.735976 & 0.42 & 648 & 14035.88 & 1.000000 \\
\hline
\end{tabular}


Table 2. Basic features of ERPs to subclasses of verbs (V) and nouns (S)

\begin{tabular}{|c|c|c|c|c|c|c|c|c|c|c|c|c|c|c|c|c|c|c|}
\hline $\begin{array}{l}n \\
0 \\
0\end{array}$ & 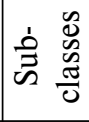 & $\mathrm{N}$ & $\begin{array}{c}\text { L } \\
\text { LP1 }\end{array}$ & $\begin{array}{c}\text { A } \\
\text { BP1 }\end{array}$ & $\begin{array}{c}\text { L } \\
\text { LP2 }\end{array}$ & $\begin{array}{c}\text { A } \\
\text { BP2 }\end{array}$ & $\begin{array}{c}\text { L } \\
\text { LP3 }\end{array}$ & $\begin{array}{c}\mathrm{A} \\
\mathrm{BP} 3\end{array}$ & $\begin{array}{c}\text { L } \\
\text { LP4 }\end{array}$ & $\begin{array}{c}\text { A } \\
\text { BP4 }\end{array}$ & $\begin{array}{c}\mathrm{L} \\
\mathrm{LN} 1\end{array}$ & $\begin{array}{c}\text { A } \\
\text { BN1 }\end{array}$ & $\begin{array}{c}\mathrm{L} \\
\mathrm{LN} 2\end{array}$ & $\begin{array}{c}\text { A } \\
\text { BN2 }\end{array}$ & $\begin{array}{c}\mathrm{L} \\
\mathrm{LN} 3\end{array}$ & $\begin{array}{c}\text { A } \\
\text { BN3 }\end{array}$ & $\begin{array}{c}\text { L } \\
\text { LN4 }\end{array}$ & $\begin{array}{c}\text { A } \\
\text { BN4 }\end{array}$ \\
\hline \multirow{3}{*}{ V } & A & 120 & 132.5 & 178.9 & 229.8 & 213.8 & 487.3 & 164.7 & 580.3 & 161.4 & 175.5 & 152.7 & 283.0 & 117.3 & 396.2 & 22.8 & 727.9 & 51.0 \\
\hline & $\mathrm{C}$ & 120 & 129.9 & 159.4 & 227.6 & 164.9 & 487.0 & 138.5 & 585.1 & 143.4 & 180.0 & 158.7 & 284.0 & 146.9 & 396.9 & 241.0 & 730.1 & 154.4 \\
\hline & $F$ & 120 & 133.7 & 180.6 & 226.3 & 183.1 & 475.0 & 160.8 & 580.8 & 9.4 & 177.9 & 3.1 & 288.4 & 8.4 & 400.3 & 209.5 & 722.8 & 36.9 \\
\hline \multirow{4}{*}{ S } & B & 120 & 132.7 & 159.0 & 234.1 & 185.9 & 482.0 & 180.5 & 579.2 & 158.7 & 175.7 & 126.6 & 290.2 & 114.4 & 403.2 & 212.4 & 723.9 & 161.6 \\
\hline & E & 120 & 133.1 & 21 & 229.7 & 203.4 & 488.1 & 190.6 & 588.3 & 145.4 & 179.7 & 115.9 & 290.6 & 89.3 & 401.5 & 171.7 & 720.7 & 162.7 \\
\hline & G & 120 & 130.8 & 180.3 & 227.2 & 185.5 & 480.5 & 182.7 & 579.9 & 141.8 & 178.3 & 141.5 & 286.6 & 142.6 & 394.6 & 208.5 & 735.4 & 189.1 \\
\hline & D & 120 & 130.6 & 178.1 & 233.7 & 197.2 & 490.1 & 175.4 & 587.1 & 137.8 & 183.4 & 135.8 & 281.5 & 125.5 & 400.5 & 191.9 & 738.7 & 155.8 \\
\hline
\end{tabular}

Subclasses of verbs: A - abstract, C - motion, F - action; Subclasses of nouns: B - abstract, E - visual, G - motor, D - animal names.

L - latencies (in ms) of positive (LP1, LP2, LP3, LP4) and negative (LN1, LN2, LN3, LN4) waves of ERPs.

A - amplitudes (in mV) of positive (BP1, BP2, BP3, BP4) and negative (BN1, BN2, BN3, BN4) waves of ERPs; Amplitude calibration: $1 \mu \mathrm{V}=40 \mathrm{mV}$.

Table 3. Interaction between ERPs to words with the same semantic attributes and a gender of participants

\begin{tabular}{|c|c|c|c|c|c|c|c|c|c|c|c|c|c|c|c|c|c|c|}
\hline 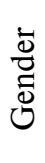 & 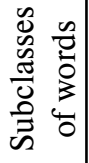 & $\mathrm{N}$ & $\begin{array}{c}\text { L } \\
\text { LP1 }\end{array}$ & $\begin{array}{c}\text { A } \\
\text { BP1 }\end{array}$ & $\begin{array}{c}\text { L } \\
\text { LP2 }\end{array}$ & $\begin{array}{c}\text { A } \\
\text { BP2 }\end{array}$ & $\begin{array}{c}\text { L } \\
\text { LP3 }\end{array}$ & $\begin{array}{c}\text { A } \\
\text { BP3 }\end{array}$ & LP4 & BP4 & LN1 & BN1 & LN2 & $\begin{array}{c}\mathrm{A} \\
\mathrm{BN} 2\end{array}$ & $\begin{array}{c}\mathrm{L} \\
\mathrm{LN} 3\end{array}$ & $\begin{array}{c}\text { A } \\
\text { BN3 }\end{array}$ & \begin{tabular}{|c|} 
L \\
LN44
\end{tabular} & $\begin{array}{c}\text { A } \\
\text { BN4 }\end{array}$ \\
\hline 1 & A & 42 & 133.5 & 135.9 & 233.8 & 135.9 & 486.2 & \begin{tabular}{|l|l|}
167.8 \\
\end{tabular} & 569.1 & \begin{tabular}{|l|l}
163.8 \\
\end{tabular} & \begin{tabular}{|l|l}
178.1 \\
\end{tabular} & \begin{tabular}{|l|l}
187.2 \\
\end{tabular} & 278.7 & 108.4 & 395.6 & 208.9 & 738.3 & $\mid 134.0$ \\
\hline 2 & A & 78 & 132.0 & 202.1 & 227.7 & 255.7 & 487.9 & 163.1 & 586.3 & 160.1 & 174.1 & 134.1 & 285.3 & 122.1 & 396.5 & 230.3 & 722.3 & 160.1 \\
\hline 1 & C & 42 & 29.5 & 157. & 36 & 120. & 84.0 & 157.3 & 59 & 198.5 & 93.4 & 183.1 & 274.5 & \begin{tabular}{|l|}
106.8 \\
\end{tabular} & 401.5 & 20 & 758. & \begin{tabular}{|l|l}
137.9 \\
\end{tabular} \\
\hline 2 & $\mathrm{C}$ & 78 & 130.2 & 160.3 & 222.7 & 188.9 & 488.6 & 128.3 & 582.4 & 113.7 & 172.7 & 145.6 & 289.1 & 168.5 & 394.4 & 242.9 & 714.9 & 163.3 \\
\hline 1 & $F$ & 42 & 39.2 & 170.8 & 33.1 & 114.5 & 468.0 & 176.4 & 85.6 & 181.1 & 180.8 & 196.4 & 276.5 & \begin{tabular}{|l|l}
118.3 \\
\end{tabular} & 403.9 & 219. & 756. & 78.6 \\
\hline 2 & $\mathrm{~F}$ & 78 & 130.8 & 185.8 & 222.6 & 220.1 & 478.8 & 152.4 & 578.2 & 132.3 & 176.3 & 129.8 & 294.8 & 133.8 & 398.4 & 204.3 & 704.7 & 168.3 \\
\hline 1 & B & 42 & 128.9 & 156.6 & 247.0 & 145.3 & 479.6 & 217.8 & 575.1 & 206.5 & 182.3 & 165.0 & 281.0 & \begin{tabular}{|l}
115.4 \\
\end{tabular} & 399.5 & 159.0 & \begin{tabular}{|l|l|}
748.2 \\
\end{tabular} & $\mid 103.8$ \\
\hline 2 & B & 78 & 134.7 & 160.3 & 227.1 & 207.8 & 483.2 & 160.4 & 581.4 & 133.0 & 172.1 & 106.0 & 295.2 & 113.9 & 405.2 & 241.1 & 710.7 & 192.7 \\
\hline 1 & $\mathrm{E}$ & 42 & 130.1 & 247.4 & 237.4 & 157.2 & 490.4 & \begin{tabular}{|l}
175.7 \\
\end{tabular} & \begin{tabular}{|l}
597.9 \\
\end{tabular} & 186.3 & 184.9 & 133.0 & 280.5 & \begin{tabular}{|l|}
52.4 \\
\end{tabular} & \begin{tabular}{|l|}
399.9 \\
\end{tabular} & 173.5 & 725.2 & 156.5 \\
\hline 2 & $\mathrm{E}$ & 78 & 134.7 & 179.5 & 225.6 & 228.2 & 486.9 & 198.6 & 583.1 & 123.4 & 176.8 & 106.6 & 296.1 & 109.3 & 402.4 & 170.8 & 718.2 & 166.1 \\
\hline 1 & $\bar{G}$ & 42 & 128.9 & 187.3 & 238.3 & \begin{tabular}{|l|l}
153.3 \\
\end{tabular} & 484.4 & 209.3 & 575.8 & 204.4 & \begin{tabular}{|l|l}
181.3 \\
\end{tabular} & \begin{tabular}{|l}
173.5 \\
\end{tabular} & 276.2 & \begin{tabular}{|l|}
144.1 \\
\end{tabular} & \begin{tabular}{|l|l|}
387.1 \\
\end{tabular} & 209.8 & \begin{tabular}{|l|l|} 
\\
\end{tabular} & 166.3 \\
\hline 2 & $\mathrm{G}$ & 78 & 131.7 & 176.5 & 221.2 & 202.8 & 478.5 & 168.3 & 582.1 & 108.1 & 176.7 & 124.2 & 292. & 141.8 & 398.6 & 207.8 & $716.3 \mid$ & 201.3 \\
\hline 1 & D & 42 & $\mid 129.8$ & 158.0 & 239.7 & 110.6 & 481.5 & 201.6 & \begin{tabular}{|l|l|}
579.8 \\
\end{tabular} & 184.8 & $\mid 197.9$ & 167.4 & 246.4 & \begin{tabular}{|l|l}
93.4 \\
\end{tabular} & 394.7 & 190.5 & 774.9 & $\mid 138.9$ \\
\hline 2 & D & 78 & 131.0 & 188.9 & 230.4 & 243.7 & 494.8 & 161.2 & 591.0 & 112.4 & 175.7 & 118.8 & 300. & 142.8 & 403.6 & 192.7 & 719.2 & 165 \\
\hline
\end{tabular}

1 - males, 2 females; Bold numbers on a grey bacground - the difference between two means is significant at the $5 \%$ level. For the others see the text to Table 2. 
verbs into three separate subclasses according to their semantics. The first subclass (abstract stimuli) consisted of abstract nouns or verbs associated with intellectual or mental activities, the second subclass (visual stimuli) contained nouns or verbs with clear visual-perceptional features, but no/few motor features, the third subclass (motor stimuli) was comprised of nouns or verbs associated with both visual-perceptional and motor features.

Kellenbach et al. ${ }^{6}$ has motivated us to undertake a similar study using Czech words. For the classification of Czech words into groups we used the same criteria as Kellenbach et al. The findings are summed up in this paper.

\section{METHODS}

The experimental paradigm, procedures, features of word stimuli and methods of data acquisition and analysis are described in detail in our paper ${ }^{10}$.

\section{RESULTS}

The results of the multivariate analysis of variance (see Tables 1) reveal the existence of significant differences in features of ERPs to three subclasses of nouns or verbs (the character of the subclasses of words is described in detail in the methodological part of the paper ${ }^{10}$, the examples are given in the appendix to it). No less significant is the interaction between semantic attributes of nouns or verbs and the gender of the participants in the experiment. However, there is no interaction between semantic attributes of words and the site of the recording electrode on the scalp.

\section{Features of ERPs to subclasses of words}

Mean latencies and amplitudes of the waves of ERPs to subclasses of words are summed up in Table 2. From it follows that basically there are only several dependent variables which significantly differ in ERPs produced by one of the subclasses of nouns or verbs. In verbs the variables are LP3, BP2 and BN3, in nouns LP2, LN1, BP1 and $\mathrm{BN} 3$.

In this context it is of interest that there are statistically significant differences in amplitudes of ERPs to nouns and verbs for which according to Kellenbach et al. ${ }^{6}$ "visual-perceptual attributes are highly salient, while motor features are not central to their semantic specification". Waves BN1, BN2 and BN3 of ERPs to motion verbs have significantly higher amplitudes than the same components of ERPs to nonmanipulable objects, in wave BP2 however, the response is the contrary (for details see Table 2).

A more detailed analysis of data in Table 2 shows that latency LP3 of ERPs to action verbs (subclass F) is shorter than the latency of the same wave of ERPs to abstract verbs (subclass A). Nouns referring to manipulable object (subclass G) evoke ERPs with shorter latency of LP2 than do abstract nouns (subclass B). On the contrary, latency of LN1 of ERPs to abstract nouns is shorter than latency of LN1 of ERPs to animal names (subclass D).

The amplitude of BP2 waves of ERPs to motion verbs (subclass C) is smaller than the amplitude of the same waves of ERPs to abstract verbs. The amplitude of BP2 component of ERPs to nonmanipulable objects (subclass E) is higher than the amplitude of the same wave of ERPs to abstract nouns. The amplitude of $\mathrm{BN} 3$ behaves quite the contrary.

\section{Gender and ERPs to subclasses of nouns and verbs}

The results of the multivariate analysis of variance (Table 1) point to an interaction between the characteristics of ERP and gender. A more detailed analysis of basic data has shown, however, that only in males can occasionally be found statistically significant differences in amplitude of wave BP1 and BN4 of ERPs to some subclasses of noun and verbs. The interaction of the two independent variables (males, females), however, comes more to the forefront in a comparison of ERPs to the same noun or verb subclass (see Table 4). The table shows that it is primarily amplitude $\mathrm{BP} 2$, the size of which depends on gender in all cases, which is significantly higher in females then in males. In the other components (BP1, BP4, BN2 and BN4) there are fewer significant differences and if they do occur, then their amplitude is higher in males than in females. In some cases, gender also affects latency of waves LP2, LN1 and LN4 of ERPs to the same noun or verb subclass - in females latencies of these waves are shorter than in males.

\section{DISCUSSION}

The findings presented in this paper demonstrate the existence of significant differences in characteristics of ERPs to three subclasses of nouns and verbs. Latency LP3 of ERPs to action verbs was shorter than the latency of the same wave to abstract verbs. In nouns the relations were similar (manipulable objects versus abstract ones), but it concerns wave LP2. The amplitude of early positive components (P1, P2) and wave BN3 was also dependent on the semantic attributes of nouns and verbs. It should be added that in our experiment some waves of ERPs to motion verbs had significantly higher amplitude than the same waves of ERPs to nonmanipulable objects.

The latter does not agree with the conclusions of Pulvermüller et al. ${ }^{14}$ and Kellenbach et al. ${ }^{6}$. Pulvermüller says that action nouns and action verbs elicit a comparable pattern of electrophysiological activity. Kellenbach et al. extended Pulvermüller's conclusion to both visual and abstract semantic attribute description. In the passage discussing his findings he says, however: "Interestingly, there is a tendency (although not supported statistically) for the grammatical class effect on N400 to be somewhat attenuated for abstract stimuli relative to visual and motor stimuli, and for the attribute effect to be reduced for verbs relative to nouns ...” This claim reduces the discrep- 
ancy between our own findings and the conclusions of Kellenbach et al. ${ }^{6}$. I admit that unification of conditions for the record of ERPs (A/D conversion rate, number of recording electrodes, etc.) and application of an identical method for assessment of their features would bring the findings from the experiments of Kellenbach et al. closer to the findings presented in this paper.

The reason why semantic attributes of nouns or verbs affect the amplitude of some waves of ERPs only in males and not in females cannot be explained at the present time. The same holds for the interaction between gender and select parameters of ERPs evoked by words with identical semantic attributes. In literature we have not found any work dealing with these problems.

\section{REFERENCES}

1. Brown WS, Marsh JT, Smith JC. (1973) Contextual meaning effects on speech evoked potentials. Behav Biol 9, 755-761

2. Damasio AR, Tranel D. (1993) Nouns and verbs are retrieved with differently distributed neural systems. Proc Natl Acad Sci USA 90, 4957-4960

3. Daniele A, Giustolisi L, Silveri CM, Colosimo C, Gainotti G. (1994) Evidence for a possible neuroanatomical basis for the lexical processing of nouns and verbs. Neuropsychologia 32, 13251342.

4. De Renzi E, Di Pellegrino G. (1995) Sparing of verbs and preserved, but ineffectual reading in a patient with impaired word production. Cortex 31, 619-636.

5. Goodglass H, Klein B, Carey P, Jones K. (1966) Specific semantic word category in aphasia. Cortex 2, 74-89.
6. Kellenbach ML, Wijers AA, Hovius M, Mulder J, Mulder G. (2002) Neural differentiation of lexico-semantic categories or semantic features? Event- related potential evidence for both. J Cogn Neurosci 14, 561-577.

7. Koenig T, Lehmann D. (1996) Microstates in language-related brain potential maps show noun-verb differences. Brain Lang 53, 169-182.

8. Miceli G, Silveri MC, Nocentini U, Caramazza A. (1988) Patterns of dissociation in comprehension and production of nouns and verbs. Aphasiology 2, 251-258

9. Molfese DL, Burger-Judisch LM, Gill LA, Golinkoff RM, HirschPasek KA. (1996) Electrophysiological correlates of noun-verb processing in adults. Brain Lang 54, 388-413.

10. Petřek J. (2004) ERPs to words - Effect of gender and site of registration electrode. Biomed. Papers 148, 145-152.

11. Pulvermüller F. (1999) Words in the brain's language. Behav Brain Sci 22, 253-336.

12. Pulvermüller F, Preissl H, Lutzenberger W, Birbaumer N. (1996) Brain rhythms of language: nouns versus verbs. Europ J Neurosci 8, 937-941.

13. Pulvermüller F, Lutzenberger W, Preissl H. (1999a) Nouns and verbs in the intact brain: Evidence from event-related potentials and high-frequency cortical responses. Cerebral Cortex 9, 497-506.

14. Pulvermüller F, Mohr B, Schleichert H. (1999b) Semantic or lexicosyntactic factors: what determines word-class specific activity in the human brain? Neurosci Lett 275, 81-84.

15. Pulvermüller F, Härle M, Hummel F. (2000) Neurophysiological distinction of verb categories. Neuroreport 11, 2789-2793.

16. Pulvermüller F, Härle M, Hummel F. (2001) Walking or Talking?: Behavioral and neurophysiological correlates of action verb processing. Brain Lang 78, 143-168

17. Silveri M, Di Betta AM. (1997) Noun-verb dissociation in braindamaged patients: Further evidence. Neurocase 3, 477-488.

18. Teyler T, Roemer RR, Harrison T, Thompson R. (1973) Human scalprecorded evoked potential correlates of linguistic stimuli. Bull Psychonom Soc 1, 333-334. 